\title{
Two-stage Turing model for generating pigment patterns on the leopard and the jaguar
}

\author{
R. T. Liu, ${ }^{1}$ S. S. Liaw, ${ }^{1}$ and P. K. Maini ${ }^{2}$ \\ ${ }^{1}$ Department of Physics, National Chung-Hsing University, 250 Guo-Kuang Road, Taichung, Taiwan \\ ${ }^{2}$ Centre for Mathematical Biology, Mathematics Institute, Oxford University, 24-29 St. Giles', Oxford OX1 3LB, United Kingdom
}

(Received 9 May 2005; revised manuscript received 12 March 2006; published 21 July 2006)

\begin{abstract}
Based on the results of phylogenetic analysis, which showed that flecks are the primitive pattern of the felid family and all other patterns including rosettes and blotches develop from it, we construct a Turing reactiondiffusion model which generates spot patterns initially. Starting from this spotted pattern, we successfully generate patterns of adult leopards and jaguars by tuning parameters of the model in the subsequent phase of patterning.
\end{abstract}

DOI: 10.1103/PhysRevE.74.011914

PACS number(s): 87.10.+e, 47.54.-r, 82.40.Ck, 05.45.-a

\section{INTRODUCTION}

The origin of pigmentation patterns on animal coats has certainly raised questions in every curious mind. In his story "How the leopard got his spots" [1], Kipling was not trying to give the question a scientific answer. He did not mean to be serious about Darwinism either when he said the zebra got his stripes pattern by standing long in the checked shadow. The law of natural selection might have already answered the why question, but to many scientists, an answer, which modern genetics obviously can not offer, to the how question would be more meaningful. One possible mechanism to explain how animals get their coat patterns was proposed by Turing [2] in 1952. By assuming there are two kinds of morphogens diffusing on a surface and interacting with each other, he showed that the distributions of the morphogens would form spatial patterns. In 1972, Gierer and Meinhardt [3], with the help of a computer, successfully generated spatial patterns of anatomical structures in hydra using the Turing model and showed that the model was consistent with the results of a number of regeneration and implantation experiments. Since then many Turing-type models described by coupled systems of reaction-diffusion equations have been used for generating patterns in mammals [4,5], fish [5-8], ladybugs [9], bacterial colonies [10-12], phyllotaxis [13,14], and many others. The text book Mathematical Biology [15] written by Murray contains many such examples, as well as analysis techniques for and properties of Turing models.

One caveat is in order. People used Turing models because these models are powerful in generating patterns, not because there is, as yet, uncontroversial evidence that patterns in biology arise as a result of a Turing reactiondiffusion process. However, evidence of morphogens-the essential ingredients of the Turing models-has emerged recently [16], and Turing structures were discovered in chemistry some 15 years ago $[17,18]$. Thus, soon the study of the Turing model and its application to biological systems may not simply be of academic interest.

Amongst the myriad of pigment patterns exhibited in the animal kingdom, the spotted pattern of the leopard holds a certain fascination. The "spotted" pattern of the leopard is actually more precisely a rosette pattern. Even without the help of the charming fable [1], it still attracts researchers [19-22] because it offers an optimal level of challenge for generating it. The same may be said for the patterns on the jaguar, which appear too complicated to be generated by a straightforward application of a Turing model.

The pigmentation patterns on leopards and jaguars change as they grow. In Figs. 1 and 2 we show pictures of a leopard and a jaguar, respectively, in three stages of development with distinct coat patterns [23]. Broadly speaking, the markings change from spots to rosettes. This pattern transformation is consistent with the results of a recent phylogenetic analysis [24]. In light of this, we construct a model in which we propose two stages of patterning. In the first stage, spots are generated in a similar manner for both the leopard and the jaguar. In the second stage, we tune model parameters to generate, separately, the patterns of the adult animal. We show that, in this way, the temporal sequence of patterns shown in Fig. 1 and Fig. 2 can be produced by our model.

\section{A TWO-STAGE MODEL}

We use the simple reaction-diffusion model adopted by Barrio et al. [25] which is essentially transformed so that it has a steady state at $(0,0)$, that is, the variables should be viewed as deviations from a steady state, rather than actual concentrations. The model has very simple nonlinear interaction terms: quadratic and cubic interactions between two morphogens $u$ and $v$ :

$$
\begin{aligned}
& \frac{\partial u}{\partial t}=D \delta \nabla^{2} u+\alpha u+v-r_{2} u v-\alpha r_{3} u v^{2}, \\
& \frac{\partial v}{\partial t}=\delta \nabla^{2} v+\gamma u+\beta v+r_{2} u v+\alpha r_{3} u v^{2},
\end{aligned}
$$

where $D$ is the ratio of the diffusion coefficient of the activator $u$ to that of the inhibitor $v$, and $\delta$ is a scaling variable which can be viewed either as the relative strength of the diffusion compared to the interaction terms, or as a measure of the length scale in the problem. It is known that $D$ has to be smaller than one to obtain spatially nonuniform stable patterns. The quadratic and cubic interaction terms favor patterns of spots and stripes, respectively [25-27]. We consider the above problem on the spatial domain of a square and impose zero flux boundary conditions. Without diffusion, the system has two stable uniform solutions. By taking $\gamma=-\alpha$, 
(a)
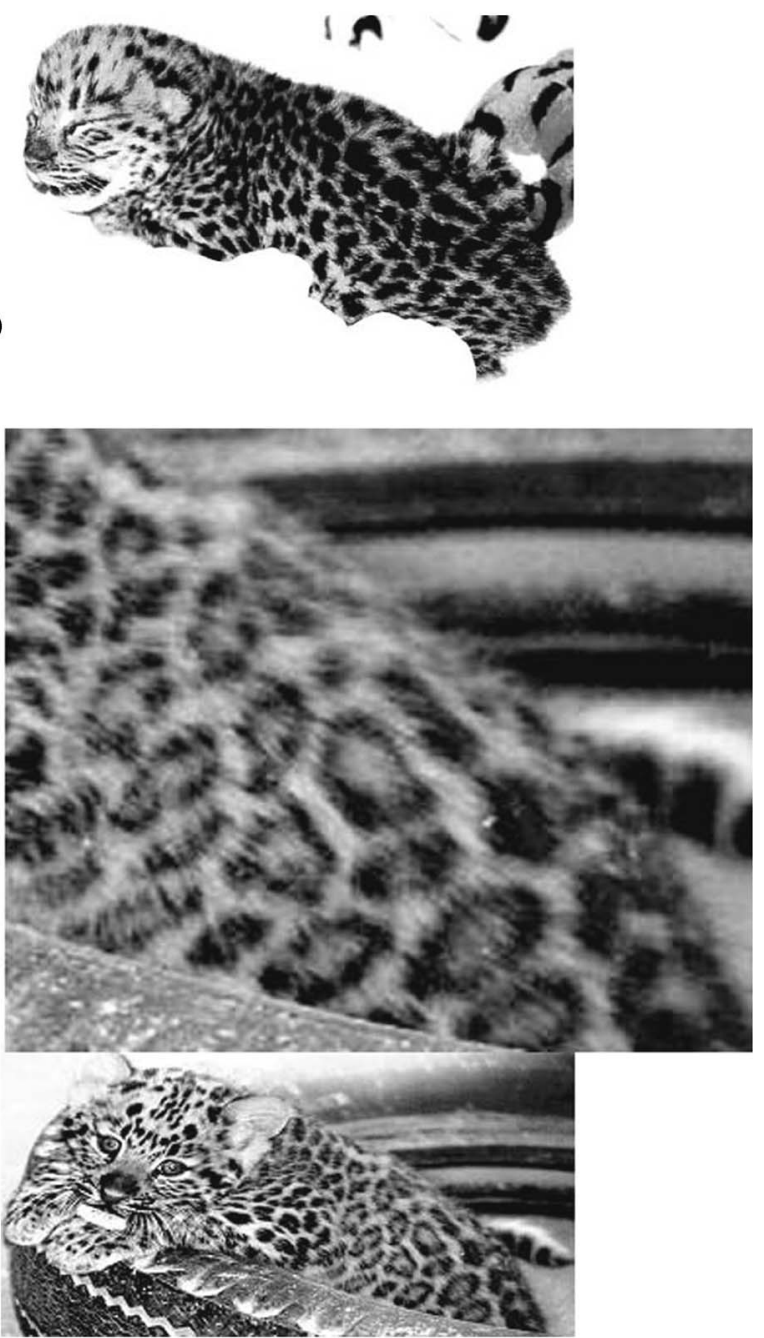

(b)

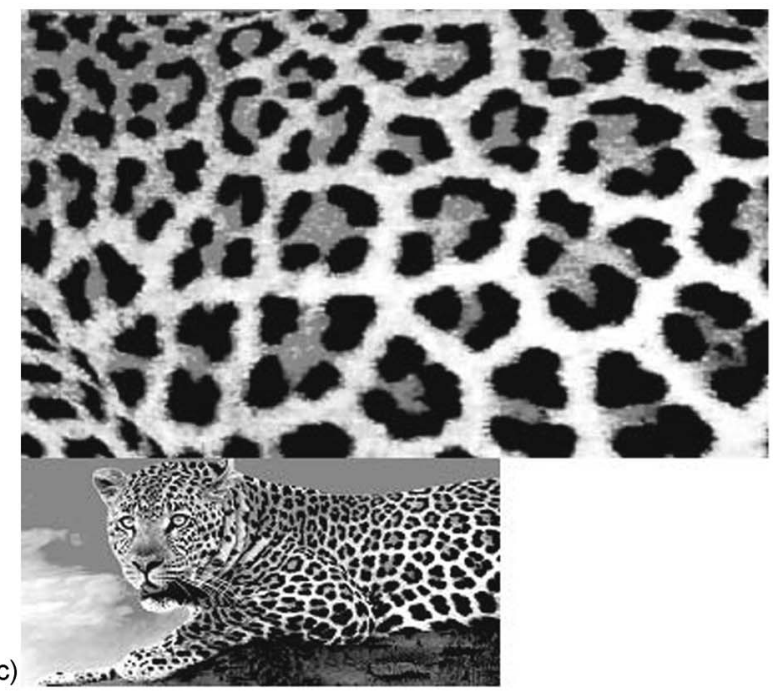

FIG. 1. Coat patterns of a leopard at different stages of growth: (a) spots (2 days), (b) rings (8 weeks), (c) rosettes (adult).

the two solutions degenerate to be simply the one $(u, v)$ $=(0,0)$ at all points in space considered.

Standard linear analysis [15] can be carried out to guide the choice of parameter values $D, \delta, \alpha, \beta, r_{2}$, and $r_{3}$, so that the model exhibits "diffusion driven" (or Turing) instability, that is, the uniform steady state is stable in the absence of diffusion but becomes unstable when diffusion is present. In the case of this model, the linearization is trivial since one simply ignores the higher order terms in Eqs. (1). As usual, we assume that the morphogen concentrations $u$ and $v$ take the form

$$
\begin{aligned}
& u(\vec{x}, t) \sim u_{0} e^{\lambda t} e^{i \vec{k} \cdot \vec{x}}, \\
& v(\vec{x}, t) \sim v_{0} e^{\lambda t} e^{i \vec{k} \cdot \vec{x}} .
\end{aligned}
$$

Imposing the zero-flux condition on the boundary (which means that morphogens do not flow out of the region considered), results in solutions with the form Eq. (2) only being admissible for a set of discrete values of $k$, which are called admissible modes. The temporal growth rate $\lambda$ can easily be found as a function of the possible modes indicated by $k$, by substituting the above solution into the linearized version of Eq. (1) yielding a dispersion relation from which one can choose parameters to allow only a small number of modes to grow in time $[\operatorname{Re}(\lambda)>0$, so that the diffusion-driven instability leads to patterns] and, in particular, to enhance any particular mode $[25,28]$. We use the following set of values for parameters to generate a stable spotted pattern on a square of $200 \times 200$ points shown in Fig. 3. The initial values of $u$ and $v$ at each point are assigned randomly between 0 and 1 .

$$
\begin{gathered}
D=0.45, \quad \delta=6, \quad \alpha=0.899, \quad \beta=-0.91, \quad r_{2}=2, \\
r_{3}=3.5 .
\end{gathered}
$$

With this set of parameters, the mode with $k=0.27$ is enhanced most and this corresponds to the spot pattern (Fig. 3). This is stage 1. We now use this as our initial distributions for $u$ and $v$ to generate, in stage 2, the patterns in the adult. The use of a spotted pattern for our initial distributions of $u$ and $v$ is based on the recent report of Werdelin and Olsson [24]. They found, regardless of which of several different phylogenies of felids is used, that flecks (small spots not organized into patterns) are the primitive pattern of the felid family. All other patterns, including rosettes (small spots organized into patterns of six or fewer spots) and blotches (irregularly shaped areas of dark on a usually lighter background), develop from it. We see from Fig. 1 and Fig. 2 [23] that the patterns on leopards and jaguars are indeed spots (flecks) in the young, which then gradually transform into, respectively, rosettes and blotches in the adult [29]. Pigmentation pattern alteration during growth is observed in a number of animals [6,30]. For example, Asai et al. have used a Turing reaction-diffusion model to simulate the skin pigmentation patterns of the zebrafish in various stages of growth by changing parameters with time [30]. Aragon et al. have also used a particular pattern as a seed for generating spatial pattern in sea urchins [28].

\section{PARAMETER SELECTION DURING GROWTH}

We start with the parameter values given in Eq. (3) and obtain the spotted pattern shown in Fig. 3. We then, in the 
(a)
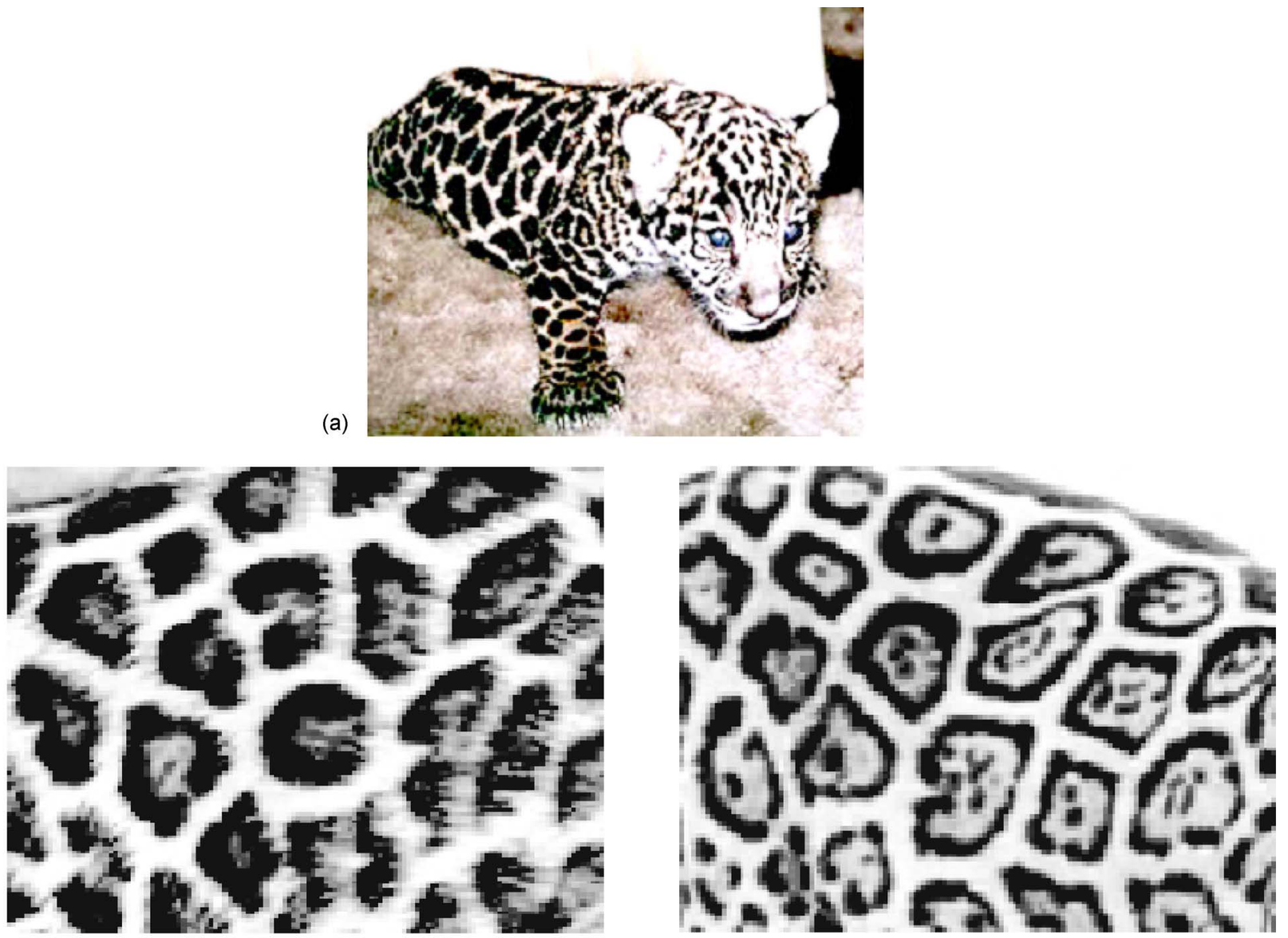

(b)

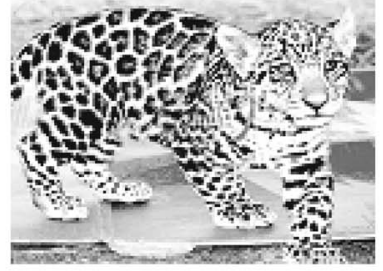

(c)

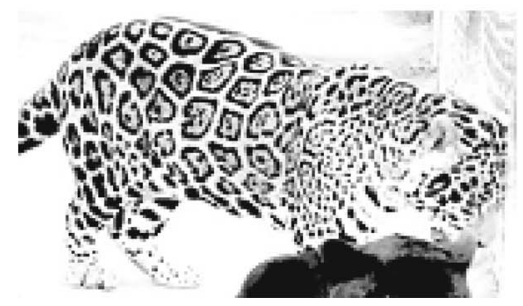

FIG. 2. (Color online) Coat patterns of a jaguar at different stages of growth: (a) spots (5 weeks), (b) irregular rings (3 months), (c) small spots enclosed by irregular broken polygons (adult).

second stage, increase $r_{2}$ and decrease both $\delta$ and $D$ in steps to generate the final adult patterns.

First we increase the value of $r_{2}$ from 2 to 7 . As stated above, the quadratic term in Eq. (1) favors spots. Increasing the value of $r_{2}$ will enhance the density of spots and increase the spatial gradients of morphogen concentration profiles. The initial spots in Fig. 3 grow darker and begin to change into rings [Fig. 4(a)]. We next make changes to $D$ and $\delta$. At this point, let us discuss the effects of parameter $D$ and $\delta$.

A general linear analysis $[15,28]$ shows that the necessary conditions for yielding Turing patterns are given by

$$
\begin{gathered}
f_{u}+g_{v}<0, \\
f_{u} g_{v}-f_{v} g_{u}>0, \\
D g_{v}-f_{u}>0,
\end{gathered}
$$

$$
\left(D g_{v}-f_{u}\right)^{2}-4 D\left(f_{u} g_{v}-f_{v} g_{u}\right)>0
$$

where $f_{u}, f_{v}\left(g_{u}, g_{v}\right)$ denote the partial derivatives of $f(g)$ with respect to $u$ and $v$. Based on these conditions we plot the $(\alpha, \beta)$ parameter space to show the Turing space-the region in which Turing patterns are predicted to occur from the linear stability analysis (Fig. 5). Our choice of $(\alpha, \beta)$ is indicated in Fig. 5.

The dispersion relation $\lambda(k)$ can be found [28] from the characteristic equation:

$$
\begin{aligned}
\lambda^{2}+ & {\left[(1+D) \delta k^{2}-\alpha-\beta\right] \lambda+D \delta^{2} k^{4}-\delta(\alpha+D \beta) k^{2} } \\
& +\alpha(\beta+1)=0
\end{aligned}
$$

To see the effects of the parameters $D$ and $\delta$, we plot in Fig. 6 the dispersion relation corresponding to several values of one parameter while keeping the others fixed. We see in general that the available Turing modes $[\operatorname{Re}(\lambda)>0]$ shift to 


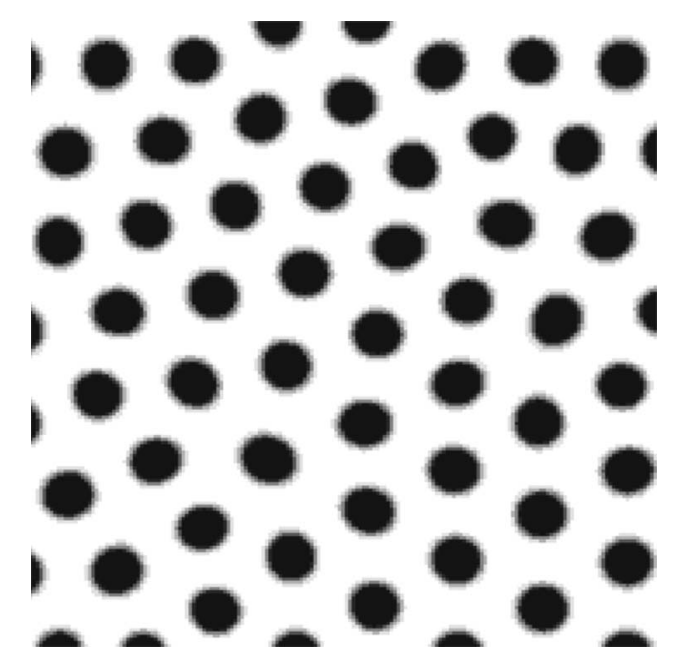

FIG. 3. Spot pattern generated by the Turing model Eq. (1). Parameters are $D=0.45, \delta=6, \alpha=0.899, \beta=-0.91, r_{2}=2, r_{3}=3.5$. The pattern is used as the initial distributions for the morphogens $u$ and $v$ in the second stage of the model.

higher wave numbers (larger $k$ ) when $\delta$ is decreased (see, for example Ref. [15]). The minimal value of wave number $k$ that can support the Turing pattern can be easily found to be inversely proportional to the square root of $\delta$ [28]. On the other hand, when $D$ is decreased, the available Turing modes increase, and all available modes are enhanced further $(\lambda$ gets larger).

With this understanding, for the leopard pattern, we force the system to switch to a higher wave number mode by decreasing $\delta$ from 6 to 3.8. We immediately see a few rings begin to break. The breaking process continues until we change the value of $D$ to a lower value $D=0.15$ to stabilize the pattern [Fig. 4(b)] [31]. For the jaguar pattern, similar steps are taken: increase $r_{2}$ first, and then decrease $\delta$, and finally decrease $D$. There are two different settings from the case of the leopard. We increase $r_{2}$ from 2 to 7 and keep it fixed at that value for a longer time than in case of the leopard so that the rings become larger and compress one another to form hexagonal shapes. Note also that an inner ring begins to appear so that every ring changes to a double ring [Fig. $7(\mathrm{a})]$. In the second step, we decrease $\delta$ to 1.8 , which is lower than the value 3.8 in the leopard case, to "break" modes. Thus the enhanced mode shifts to one with a higher wave number according to the dispersion relation shown in Fig. 8. The inner ring breaks first. When the inner rings are broken completely and some outer rings begin to break, we decrease $D$ (to 0.15 ) to stabilize the final pattern [Fig. 7(b)] [31].

\section{COMPARISON WITH REAL PATTERNS}

Coat patterns of a typical leopard at three stages of growth are shown in Fig. 1 [23]. Roughly speaking, the pattern grows from spots to rings and then to rosettes. Our simulation aims to follow this temporal sequence and indeed it successfully generates the sequence of patterns [Fig. 3, Figs. 4(a) and 4(b)]. Previous simulations using a one-stage Turing

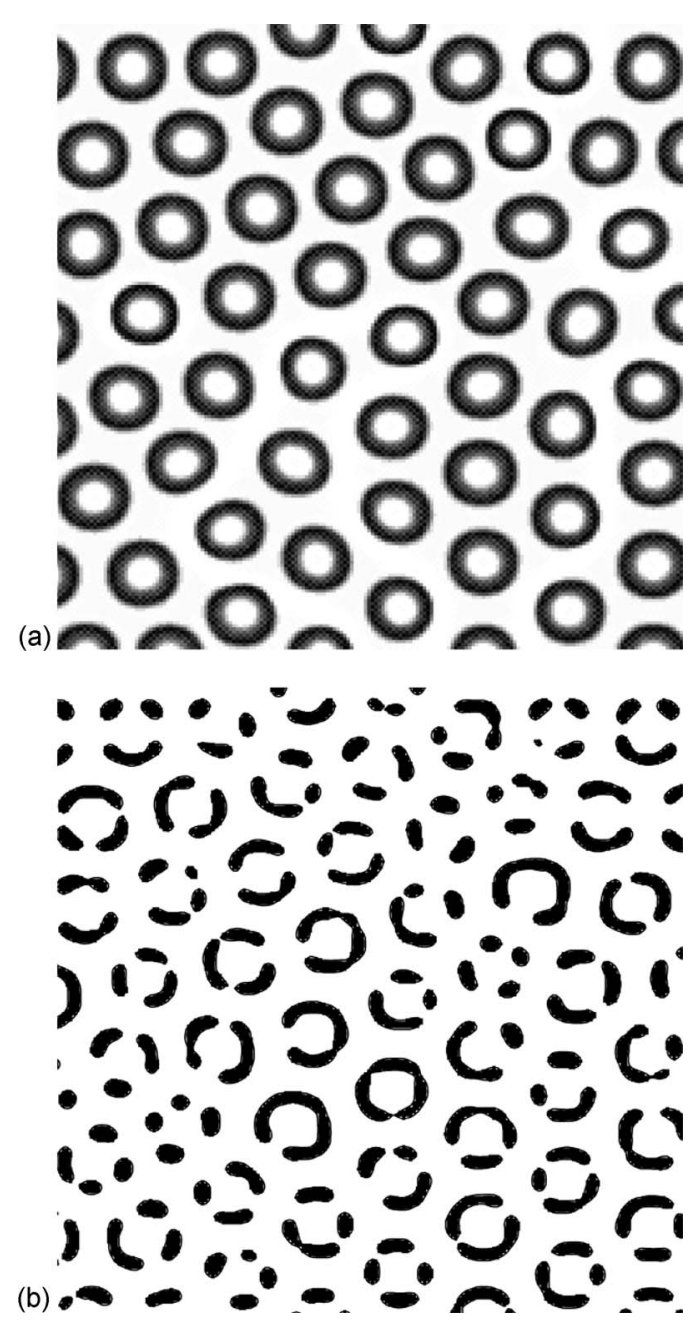

FIG. 4. Simulated patterns corresponding to the case of the leopard in the second stage. Starting from the spot pattern (Fig. 3), we increase $r_{2}$ from 2 to 7 to obtain (a), and then decrease $\delta$ from 6 to 3.8 to obtain (b), which is then stabilized by decreasing $D$ from 0.45 to 0.15 .

model $[19,20,22]$ might have produced final rosette patterns similar to the patterns of real leopards, but they left the process of the sequential development of pattern unanswered.

Our results for the pigment patterns of the jaguar [Fig. 3, Figs. 7(a) and 7(b)] compare well with those actually observed (Fig. 2). The patterns in the adult (small spots enclosed by irregular broken polygons) are particularly difficult to generate using a one-stage Turing model. Even if it is possible, it still fails to explain the temporal series of patterns observed during the growth of the animal. Our model suggests that it would be easier to obtain a complicated pattern in steps, and at the same time, one can capture key aspects of the temporal development.

Note that our simulations are consistent with the fact that the number of rosettes or patches on the adult leopard or jaguar is roughly the same with the number of spots on younger animals. This is different from the case of angelfish where the number of stripes increases as the fish grows [6]. 


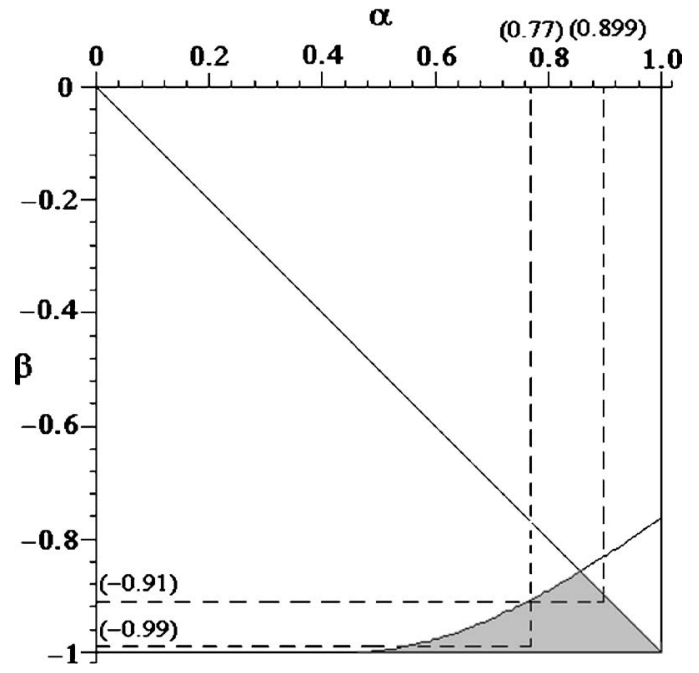

FIG. 5. The Turing space of the model described by Eq. (1) $(D=0.45)$. The shaded region is where linear analysis predicts the possibility of Turing patterns. Three different sets of parameters $(\alpha, \beta)=(0.899,-0.91),(0.77,-0.91),(0.77,-0.99)$ used in simulations are indicated by the crossed points of dashed lines.

\section{DISCUSSION}

The choice of the parameters for producing patterns in different steps for either the leopard or jaguar is not unique. There are wide ranges of values of the parameters that we can use to obtain spotted patterns, and any of which can, in principle, be used as initial distributions to generate patterns

(a)

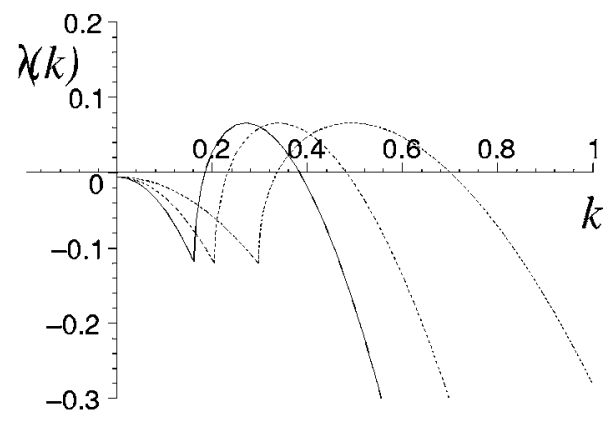

(b)

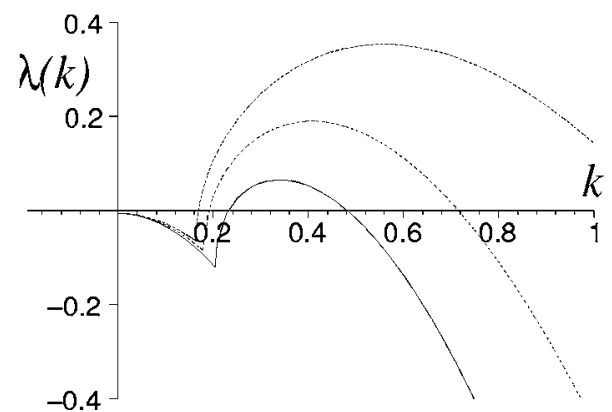

FIG. 6. Dispersion relation $(\alpha=0.899, \beta=-0.91)$ for (a) $D$ $=0.45, \delta=6$ (solid line), 3.8 (dotted line), 1.8 (dashed line). When $D$ is fixed, the growing modes shift to high wave numbers as $\delta$ decreases; (b) $\delta=3.8, D=0.45$ (solid line), 0.3 (dotted line), 0.15 (dashed line). When $\delta$ is fixed and $D$ decreases, all available modes are enhanced further.

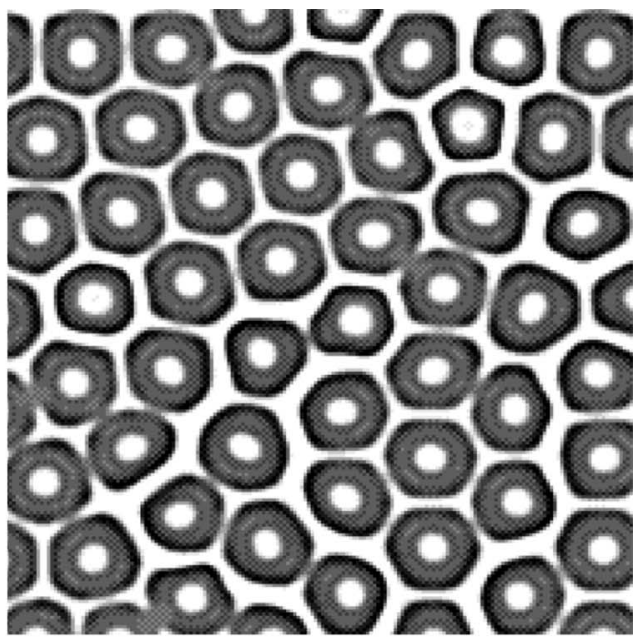

(a)

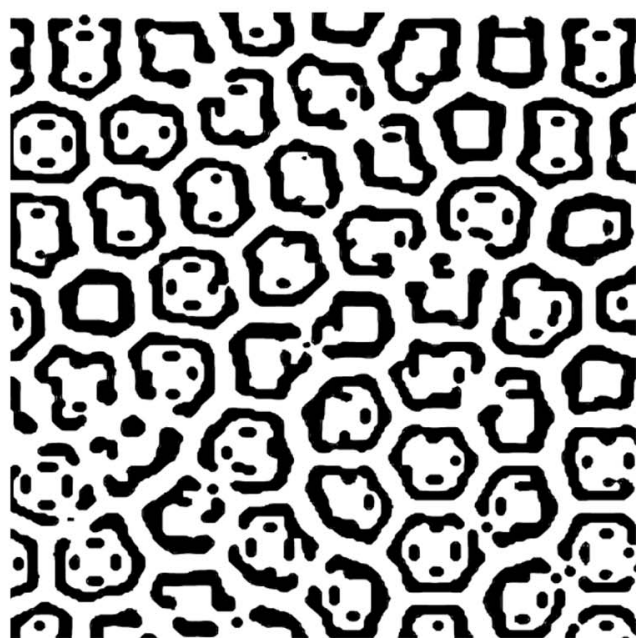

FIG. 7. Simulated patterns for the jaguar in the second stage. Starting from the spot pattern (Fig. 3), we increase $r_{2}$ from 2 to 7 for a sufficiently long time to obtain the pattern in (a). Next we decrease $\delta$ from 6 to 1.8 to obtain the pattern (b), which is then stabilized by decreasing $D$ from 0.45 to 0.15 .

of the adult leopard and jaguar as long as the subsequent variations can be adjusted accordingly. For example, two other choices for parameters $\alpha$ and $\beta$ in the Turing space are $(0.77,-0.91)$ and $(0.77,-0.99)$ (Fig. 5). We are able to obtain similar patterns shown in Figs. 4 and 7. This is very

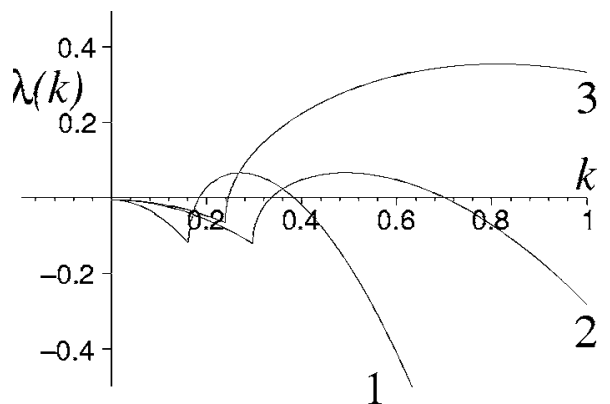

FIG. 8. Dispersion relations for the parameters used in sequence in simulating the jaguar patterns. $1: D=0.45, \delta=6 \rightarrow 2: D=0.45, \delta$ $=1.8 \rightarrow 3: D=0.15, \delta=1.8$. 


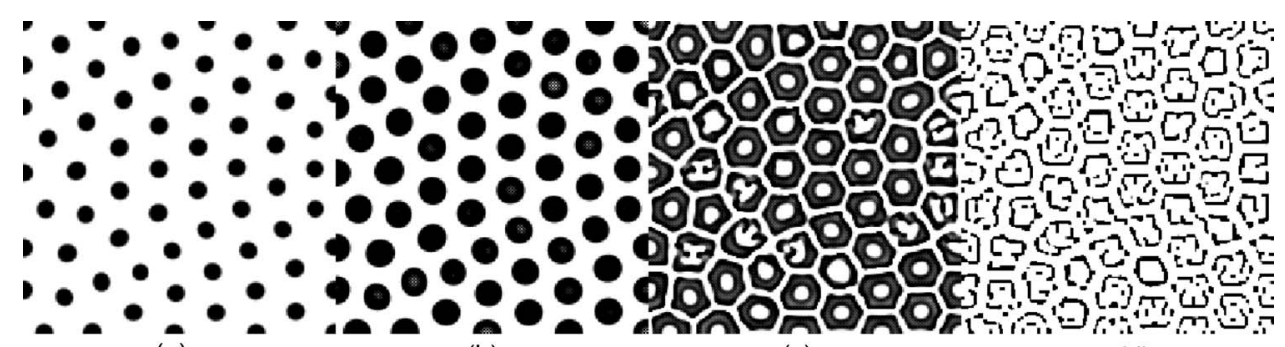

(a)

(b)

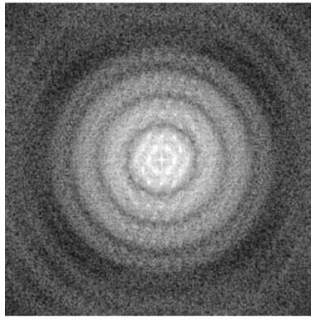

(e)

(f) (c)

(d)

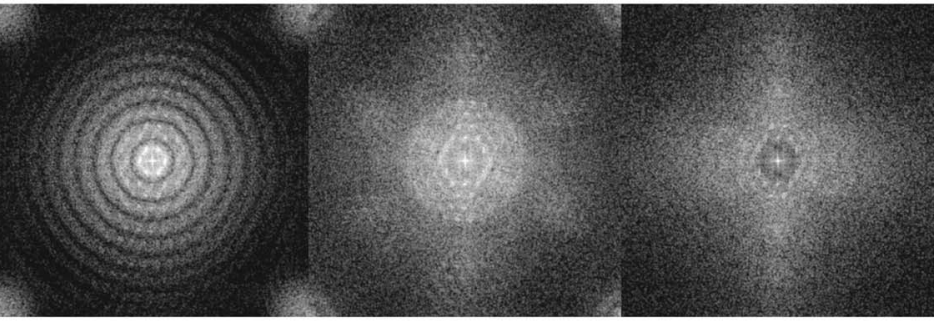

(g)

(h)

FIG. 9. Simulation patterns of the jaguar at four sequential steps (a)-(d) and the corresponding spectra of two-dimensional Fourier transform (e)-(h). The dimensions of the spatial pattern are $200 \times 200$ pixels. Using pixel as the unit of length, the distance between two spots in patterns (a) and (b) is about 25 on average. Thus the first conspicuous frequency in the power spectrum is about 0.04 which appears in (e) as the smallest white ring in the middle. The smallest white ring in (g) has a radius of about 0.08 . This can be understood since the average distance between bands of the rings in (c) is about one half of the distance between spots in (a) or (b). The frequency range is from 0 (center) to 0.5 (edge) in (e)-(h). We have taken log values of the intensity to increase contrasts of the spectra (e)-(h).

important because the requirement of fine tuning in parameters can hardly explain why the general patterns for these animals are robust. In general, robustness is difficult to obtain for global patterning mechanisms. The work of Dillon et al. [32] and Crampin et al. [33] has shown how modifying the Turing system through controlling the boundary conditions or incorporating domain growth can greatly increase the robustness of certain patterns. In the present study, the crucial point for generating patterns of the felid family by a Turing model is by use of the two-stage growth process in which the spotted pattern is generated in the first stage. The final patterns are determined by suitable changes of parameters and the timing of their changes in the second stage. In particular, the timing of the last decrease of parameter $D$ plays an important role. In the case of jaguar, when $\delta$ is decreased, the inner rings normally break into four small spots and some of these spots gradually disappear. A timely decrease of $D$ produces our final pattern [Fig. 7(b)]. Due to the complexity of the parameter space, it is formidable for the present to map out all the possible ranges of parameters that can yield desired patterns. Moreover, in the second stage process we are dealing with spatially dependent patterns and so to fully understand the effects of parameter values would require a stability analysis in a highly nonlinear regime. This could only be done numerically. However, as we see below, the general intuition we have gained on how a certain parameter affects the system in the linear regime seems to hold in this much more complex situation.

In the second stage of the simulation, we change the parameters $r_{2}, \delta$, and $D$ sequentially from one value to another. Although we do not have precise evidence that parameters change in the system, we are motivated to change the diffusion coefficient since it sets the overall scale of the system and this changes as the individual grows. Since the growth of an animal is a continuous process, the change of $\delta$ is more likely to change continuously as well. In view of this, we have further adopted two continuous transition processes for parameters $r_{2}, \delta$, and $D$ changing from one value to another. The first is a linear function for the change of a parameter $g$ from $g_{i}$ to $g_{f}[30]$ :

$$
g(t)=g_{i}+c\left(g_{f}-g_{i}\right)
$$

where $c$ is the changing rate of the parameter $g$ during the changing period. Another more smooth function at both ends of the changing period is given by the logistic growth function:

$$
g(t)=\frac{g_{f}}{1+e^{-c t}\left(g_{f} / g_{i}-1\right)} .
$$

Again, the value of $c$ governs the changing rate. We found that it is possible to find a range of possible values of $c$ for each parameter $r_{2}, \delta$, and $D$ so that the results obtained in Sec. III hold. Namely, we can obtain similar final patterns for either the leopard or the jaguar with those using sudden changes of parameters. For example, for the case of jaguar pattern Fig. 7(b), we use Eq. (7) to change $\delta$ from 6 to approach 1.8. A similar pattern to Fig. 7(b) is obtained when the changing rate $c$ is greater than 1.0. We are currently working on the details of these simulations and their analysis.

To gain some insights to the patterns, we compute the power spectra of the simulation patterns using the twodimensional Fourier transform. In Fig. 9 we show our simulation patterns of the jaguar at four consecutive steps together with their two-dimensional power spectra. We see 


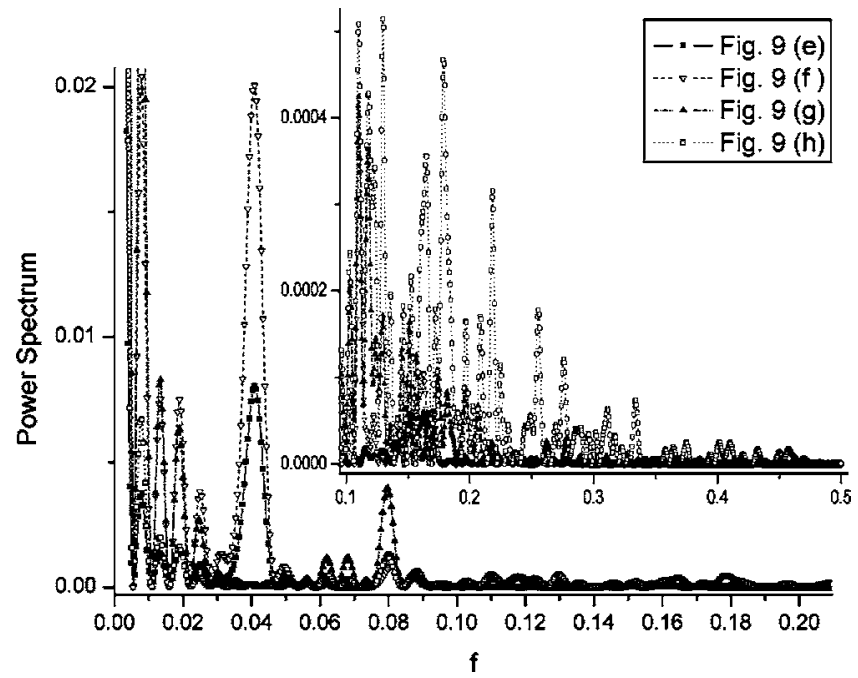

FIG. 10. Intensity of the power spectrum of Figs. 9(e)-9(h) along the horizontal line from center to one edge. Two frequency ranges $[0,0.2][0.1,0.5]$ are plotted separately to show details.

that, as the spatial patterns go from small spots to larger spots, then rings, and finally broken rings, the high frequency modes (white regimes with large radii to center) are gradually enhanced while the lowest conspicuous frequency mode [the white ring with smallest radius in Fig. 9(e)] disappears at the third step [Fig. $9(\mathrm{~g})]$. It is even clearer from Fig. 10 where we plot one-dimensional power spectra by taking intensity along the horizontal line through the center of every two-dimensional frequency domain in Fig. 9. In the first step, when the spatial pattern consists of spots, there is a peak at low frequency 0.041 in the power spectrum. When the parameter $r_{2}$ is increased in the second step, this peak is enhanced and some high frequency modes begin to appear. For the rings pattern [Fig. 9(c)], this low frequency peak disappears, consistent with Fig. 6(a) where we see the effect of decreasing $\delta$. The most enhanced frequency is now 0.081 which is about two times the value for the case of the spot pattern. This can be understood because the average distance between the bands of the rings is about one half of the distance between spots. In our final pattern for the jaguar we have some broken rings which means more high frequency modes appear in the power spectrum.

We can directly see the relation between a spatial pattern and its frequency modes by comparing the result of the inverse Fourier transformation of the truncated frequency domains with the original pattern. Take the pattern of the third step shown in Fig. 9(c) for example. Truncating the frequency at the value 0.061 [Fig. 11(a)], the resultant pattern [Fig. 11(b)] of the higher frequency part matches the original pattern [Fig. 9(c)] very closely, while that of lower frequency part [Fig. 11(c)] can be hardly recognized to be related to the original pattern. On the other hand, the spot pattern [Fig. 9(a)] can be reproduced almost completely using a low-pass filter.

\section{CONCLUSION}

A recent phylogenetic analysis showed that flecks are the primitive pigmentation pattern of the felid family and all

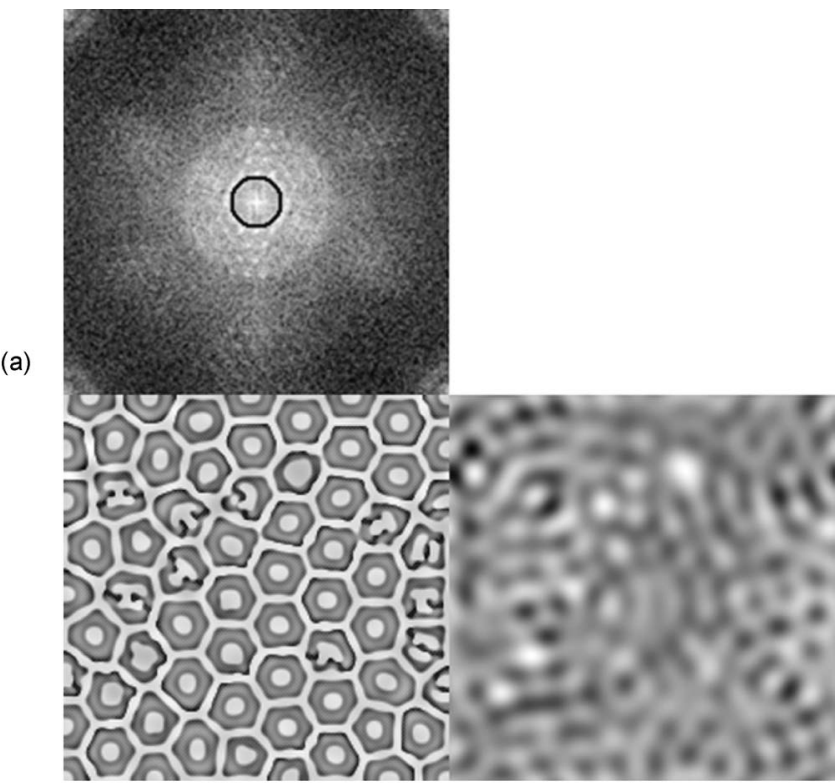

(b)

(c)

FIG. 11. Truncating the power spectrum of Fig. $9(\mathrm{~g})$ at frequency 0.061 [black circle shown in (a)], the inverse Fourier transform of the high frequency part (b) reproduces the original spatial pattern [Fig. 9(c)] well, while the low frequency part (c) has little resemblance to the original one.

other patterns, including rosettes and blotches, develop from it. The development of the coat makings of the leopard and jaguar is consistent with this result. Based on this result, we took a simple Turing model and generated patterns at different growth stages of the leopard and jaguar by tuning parameters of the model in steps. We first generated the spotted pattern for the young leopard and jaguar. In the second stage, three parameters were changed in sequence to enhance, to shift, and to stabilize spatial modes consistent with the pattern transformation observed during the growth of the animals. The rosette patterns on adult leopards were obtained in three steps, while the more complicated patterns on adult jaguars were obtained similarly with a different set of parameters.

Turing models have been demonstrated previously in many examples to generate patterns similar to those observed in nature. However, few studies address the pattern changes during growth. In this article we have successfully used a simple Turing model to generate the temporal evolution of coat markings in the leopard and jaguar at different stages of growth by tuning the parameters of the model during growth. It is not unreasonable to propose that parameters of the model would change during growth. Note that although we have not explicitly included growth of the domain in this study, the parameter $\delta$ is inversely related to domain scale, so we would expect that an increase in domain size would correspond to a decrease in this parameter.

In conclusion, we have shown the ability of a Turing reaction-diffusion model to exhibit a temporal sequence of spatial patterning that is consistent with some examples in nature. This lends further support to the role of morphogens in developmental biology but of course does not prove 
it. To extend this work further requires the experimental identification of the morphogens in this context.

\section{ACKNOWLEDGMENTS}

The work is partially supported by National Science
Council of Taiwan under Grant Number NSC92-2112M005-005 and the National Center for Theoretical Science of Taiwan. R.T.L. acknowledges the hospitality of the Centre for Mathematical Biology, Mathematical Institute, Oxford.
[1] Rudyard Kipling, Just So Stories, illustrated by B. Moser (William Morrow and Company New York, 1996).

[2] Alan Turing, Philos. Trans. R. Soc. London, Ser. B 237, 37 (1952).

[3] A. Gierer and H. Meinhardt, Kybernetik 12, 30 (1972).

[4] J. B. L. Bard, J. Theor. Biol. 93, 363 (1981).

[5] J. D. Murray, Philos. Trans. R. Soc. London, Ser. B 295, 473 (1981); , J. Theor. Biol. 88, 161 (1981).

[6] S. Kondo and R. Asai, Nature (London) 376, 765 (1995).

[7] T. Hofer and P. K. Maini, Nature (London) 380, 678 (1996).

[8] C. Varea, J. L. Aragón, and R. A. Barrio, Phys. Rev. E 56, 1250 (1997).

[9] S. S. Liaw, C. C. Yang, R. T. Liu, and J. T. Hong, Phys. Rev. E 64, 041909 (2001).

[10] E. Ben-Jacob, O. Shocher, A. Tenenbaum, I. Cohen, A. Czirok, and T. Vicsek, Nature (London) 368, 46 (1994).

[11] R. Tyson, S. R. Lubkin, and J. D. Murray, Proc. R. Soc. London, Ser. B 266, 299 (1999).

[12] M. Matsushita, J. Wakita, H. Itoh, K. Watanabe, T. Arai, T. Matsuyama, H. Sakaguchi, and M. Mimura, Physica A 274, 190 (1999).

[13] H. Meinhardt, Models of Biological Patterns Formation (Academic Press, New York, 1982).

[14] S. S. Liaw, Phys. Rev. E 57, 4589 (1998).

[15] J. D. Murray, Mathematical Biology: II: Spatial Models and Biomedical Applications (Springer-Verlag, Berlin, 2003).

[16] Y. Chen and A. F. Schler, Nature (London) 411, 607 (2001).

[17] Q. Ouyang and H. L. Swinney, Nature (London) 352, 610 (1991).

[18] P. De Kepper, J. Boissonade, and I. R. Epstein, J. Phys. Chem. 94, 6525 (1990).

[19] G. Turk, Comput. Graphics 25, 289 (1991).

[20] A. J. Koch and H. Meinhardt, Rev. Mod. Phys. 66, 1481 (1994).
[21] M. Walter, A. Fournier, and M. Reimers, Clonal Mosaic Model for the Synthesis of Mammalian Coat Patterns, in Proceedings of the 28th Annual Conference on Computer Graphics, 1998 (ACM Press, New York, 2001), pp. 82-91.

[22] K. J. Painter, P. K. Maini, and H. G. Othmer, J. Math. Biol. 41, 285 (2000).

[23] Courtesy of the Feline Conservation Center of Exotic Feline Breeding Compound (EFBC/FCC), http://www.cathousefcc.org/index.html

[24] Lars Werdelin and Lennart Olsson, Biol. J. Linn. Soc. 62, 383 (1997).

[25] R. A. Barrio, C. Varea, J. L. Aragón, and P. K. Maini, Bull. Math. Biol. 61, 483 (1999).

[26] B. Ermentrout, Proc. R. Soc. London, Ser. A 434, 413 (1991).

[27] B. N. Nagorcka and J. R. Mooney, IMA J. Math. Appl. Med. Biol. 9, 249 (1992).

[28] J. L. Aragón, M. Torres, D. Gil, R. A. Barrio, and P. K. Maini, Phys. Rev. E 65, 051913 (2002).

[29] In Ref. [24], the pigmentations patterns on leopards and jaguars are characterized both by rosettes. However, judging from Fig. 2, the jaguar patterns look like a mixture of rosettes and blotches.

[30] Rihito Asai, Emiko Taguchi, Yukari Kume, Mayumi, and Shigeru Kondo, Mech. Dev. 89, 87 (1999).

[31] In our simulation, the final pattern appears in a few hundreds time steps. We have left the simulation go on for another hundred thousands steps. Although the final pattern keeps changing in details, the general feature of the pattern remains the same.

[32] R. Dillon, P. K. Maini, and H. G. Othmer, J. Math. Biol. 32, 345 (1994).

[33] E. J. Crampin, E. A. Gaffney, and P. K. Maini, Bull. Math. Biol. 61, 1093 (1999). 